\title{
ANÁLISE DO DUPLO PRODUTO DURANTE RECUPERAÇÃO PÓS-ESFORÇO FÍSICO MÁXIMO DE TABAGISTAS
}

Rebeca Nunes Silva ${ }^{1}$, Dionei Ramos ${ }^{1}$, Camila Aguiar Santiago ${ }^{1}$, Juliana Souza Uzeloto ${ }^{1}$, Fabiano Francisco de Lima ${ }^{1}$, Juliana Tiyaki Ito ${ }^{2}$, Ercy Mara Cipulo Ramos ${ }^{1}$

${ }^{1}$ Universidade Estadual Paulista - FCT/UNESP, Departamento de Fisioterapia, Presidente Prudente, SP. ${ }^{2}$ Universidade de São Paulo - USP, Departamento de Fisioterapia, São Paulo, SP. e-mail: rebecafisio12@hotmail.com - Órgão de fomento: Fundação de Amparo à Pesquisa do Estado de São Paulo - FAPESP.

\section{RESUMO}

A grande quantidade de substâncias tóxicas presentes no tabaco faz com que o seu uso cause importantes alterações hemodinâmicas, como o aumento da frequência cardíaca (FC) e pressão arterial (PA), que estão intimamente ligadas ao duplo produto (DP). Devido à sua grande relevância, o objetivo do trabalho foi comparar o comportamento do DP em tabagistas e não tabagistas durante os cinco primeiros minutos de recuperação pós-esforço físico máximo. Vinte e dois indivíduos participaram do estudo. Todos foram classificados como sedentários e apresentaram função pulmonar normal, atestada por espirometria. Os indivíduos foram submetidos a um teste progressivo exaustivo em esteira. Após o teste, verificou-se a FC e PA no primeiro, terceiro e quinto minuto de recuperação. Ao comparar o DP, não foi observada diferença estatística entre os grupos. No entanto, o fato pode ser explicado pelo período de 12 horas de abstinência tabagística do grupo tabagista.

Palavras-chave: tabagismo, frequência cardíaca, pressão arterial, teste ergométrico de esteira, exercício aeróbico.

\section{ANALYSIS OF DOUBLE PRODUCT DURING RECOVERY AFTER MAXIMUM PHYSICAL EFFORT OF SMOKERS}

\begin{abstract}
A large amount of toxic substances present in tobacco causes significant hemodynamic changes, such as increased heart rate (HR) and blood pressure (BP), which are closely linked to the double product (DP). Due to its great importance, the objective was to compare the behavior of DP in smokers and nonsmokers during the first five minutes of recovery after maximum physical exertion. Twenty two subjects participated in the study. All were classified as sedentary and had normal lung function, attested by spirometry. The subjects underwent an exhaustive progressive treadmill test. After testing, it was found to HR and BP in the first, third and fifth minute of recovery. When comparing the DP, no statistical difference between groups was observed. However, the fact can be explained by the 12-hour period of abstinence smoking of the smokers. Keywords: smoking, heart rate, arterial pressure, treadmill exercise test, aerobic exercise.
\end{abstract}




\section{INTRODUÇÃO}

Sendo considerada a principal causa de morte evitável no mundo - segundo a Organização Mundial da Saúde -, o tabagismo é um importante problema de saúde pública que acomete aproximadamente um sétimo da população mundial $^{1}$. Deste modo, o tabagismo apresenta um número de óbitos maior do que a soma de várias outras causas evitáveis $^{2,3}$. Isso se dá pelo fato de o tabaco apresentar mais de 4720 substâncias tóxicas, que são inaladas no momento da combustão $^{4}$.

Assim, como se sabe, após fumar, o tabagista apresenta um aumento da frequência cardíaca $(F C)^{5}$. Segundo Hollmann et al. ${ }^{6}$, ao fumar um único cigarro, a FC do tabagista é elevada de 10 a 20 batimentos por minuto (bpm) e esse efeito só pode ser revertido após 15 a 45 minutos de abstinência $^{6,7}$.

A vasoconstrição causada pela nicotina presente no cigarro ${ }^{8,9}$ é um fator gerador do aumento da pressão arterial $(P A)^{9}$. Por esse fato, o tabagismo é um dos principais fatores de risco para a hipertensão arterial e outras doenças cardiovasculares ${ }^{10}$.

As medidas pressóricas sistólica e diastólica, mesmo quando observadas durante 24 horas de um dia normal, sem abstinência do cigarro, se apresentam mais elevadas em tabagistas, quando comparados a não tabagistas ${ }^{5}$, e embora não existam evidências que comprovem que o cigarro está associado à hipertensão crônica, sabe-se que ele está relacionado ao aumento agudo da resistência vascular periférica e, portanto, da $P^{11}$.

A FC e a PA, isoladamente, fornecem informações importantes sobre o nível de aptidão física e adaptação a cargas de exercício. Mas, além disso, essas variáveis permitem o cálculo de uma terceira variável: o duplo produto (DP) ${ }^{12}$.

O DP é o produto da $\mathrm{FC}$ e da pressão arterial sistólica (PAS). Esta variável permite avaliar o consumo de oxigênio do miocárdio ${ }^{13}$ e é considerado o melhor método não invasivo com essa finalidade. Assim, o DP se trata de uma variável intimamente relacionada com a segurança da atividade física, já que fornece subsídios adicionais à manipulação de sua intensidade absoluta e relativa, além de permitir que avalie o trabalho do miocárdio durante o repouso ou durante esforços físicos aeróbicos, já que está relacionada com 0 consumo de oxigênio ${ }^{14}$.

Durante o exercício físico aeróbico, há um aumento da atividade simpática no sistema nervoso central, que faz com que aumente a FC. Os metabólitos musculares liberados durante o exercício promovem vasodilatação e diminuição da resistência vascular periférica e, além disso, durante o 
exercício, também é observado um aumento da $\mathrm{PAS}^{15}$. Esses aumentos hemodinâmicos ainda permanecem por um tempo após o esforço físico e, em consequência disso, o DP também se encontra elevado durante um período pós-esforço físico. No entanto, ainda não se sabe, em relação à recuperação pósesforço físico, como se comporta o DP em indivíduos tabagistas.

Assim, esse estudo teve como objetivo comparar o comportamento do DP em tabagistas e não tabagistas durante os cinco primeiros minutos de recuperação pósesforço físico máximo.

\section{MÉTODOS}

Foram inclusos no estudo 22 indivíduos, sendo 11 do grupo de tabagistas (GT) e 11 do grupo de não tabagistas, denominado grupo controle (GC). Todos os participantes foram instruídos a respeito dos métodos de avaliação e assinaram o termo de consentimento livre-esclarecido. $O$ estudo foi aprovado pelo comitê de ética em pesquisa da FCT-Unesp, campus de Presidente Prudente. Processo no 18/2011.

Inicialmente, todos foram avaliados quanto ao nível de atividade física, pelo International Physical Activity Questionnaire (IPAQ), função pulmonar, através da espirometria, e os indivíduos do GT foram questionados quanto à carga tabagística (cigarros/dia e anos que fuma). Os integrantes do GT foram orientados a permaneceram em abstinência do cigarro por 12 horas, para a realização das avaliações.

Posteriormente, ambos os grupos foram submetidos a um teste progressivo exaustivo em esteira, seguindo o protocolo de Bruce. Após o teste, foram verificados os sinais vitais (FC e PA) no primeiro, terceiro e quinto minuto de recuperação.

Os dados foram analisados pelo programa estatístico GraphPadPrism 5. Para avaliar a normalidade dos dados foi realizado o teste de Shapiro-Wilk. Para a comparação entre os grupos foi utilizado o teste $t$ não pareado para os dados normais e teste de Mann-Withney para os dados não normais. $\mathrm{O}$ nível de significância utilizado foi de $p<0.05$.

\section{RESULTADOS}

Os participantes do GC e GT apresentaram média de idade de

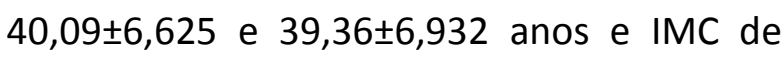
$26,89 \pm 5,624$ e $27,45 \pm 4,677 \mathrm{~kg} / \mathrm{m}^{2}$, respectivamente. Quanto ao nível de atividade física, todos foram classificados como irregularmente ativos. Os indivíduos tabagistas obtiveram média de anos/maço de $35,30 \pm 20,57$. Todos apresentaram função pulmonar normal (CVF (\%): 108,6 $\pm 12,80$ e

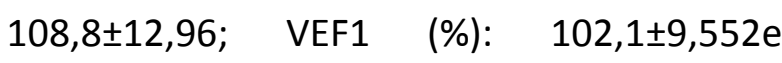
99,75 $\pm 12,79$; VEF1/CVF: 79,95 $\pm 5,054$ e 78,42 $\pm 6,693)$. Esses resultados da caracterização da amostra estão disponíveis na Tabela 1. 
Tabela 1. Caracterização da amostra

\begin{tabular}{lcc}
\hline & $\begin{array}{c}\text { Grupo Controle } \\
(\mathbf{n}=\mathbf{1 1})\end{array}$ & $\begin{array}{c}\text { Grupo tabagista } \\
(\mathbf{n}=\mathbf{1 1})\end{array}$ \\
\hline Idade (anos) & $40,09 \pm 6,625$ & $39,36 \pm 6,932$ \\
IMC (kg/m $\left.{ }^{2}\right)$ & $26,89 \pm 5,624$ & $27,45 \pm 4,677$ \\
COex (ppm) & $1,182 \pm 1,250$ & $5,545 \pm 3,142$ \\
Cigarros/dia & - & $26,82 \pm 10,55$ \\
Anos que fuma & - & $25,09 \pm 7,752$ \\
Anos/maço & - & $35,30 \pm 20,57$ \\
CVF (\%) & $108,6 \pm 12,80$ & $108,8 \pm 12,96$ \\
VEF1 (\%) & $102,1 \pm 9,552$ & $99,75 \pm 12,79$ \\
VEF1/CVF (\%) & $79,95 \pm 5,054$ & $78,42 \pm 6,693$ \\
\hline
\end{tabular}

Legenda: IMC: Índice de massa corporal; COex: Monóxido de carbono no ar exalado; ppm: partes por milhão; CVF: Capacidade vital forçada; VEF1: Volume expirado no primeiro segundo; VEF1/CVF: Relação entre VEF1 e CVF.

Ao comparar o duplo produto (DP) entre os grupos, não foi observada diferença estatística. O GT e o GC apresentaram um DP de $20223 \pm 5702$ e $22560 \pm 5208(p=0,2372)$, $14308 \pm 4149$ e $15609 \pm 2951 \quad(p=0,2003)$ e
$11982 \pm 2794$ e $13064 \pm 2441 \quad(p=0,2122)$ $\mathrm{mmHg} / \mathrm{bpm}$ no primeiro, terceiro e quinto minuto de recuperação pós-esforço físico máximo, respectivamente. Esses valores podem ser melhor visualizados na Tabela 2.

Tabela 2. Valores de pressão arterial sistólica, frequência cardíaca e duplo produto, de indivíduos tabagistas e não tabagistas no período de recuperação pós esforço máximo.

\begin{tabular}{|c|c|c|c|}
\hline & $\begin{array}{c}\text { Grupo Controle } \\
(n=11)\end{array}$ & $\begin{array}{c}\text { Grupo tabagista } \\
(n=11)\end{array}$ & Valor de $p$ \\
\hline \multicolumn{4}{|c|}{ PAS (mmHg) } \\
\hline $1^{\prime}$ & $170,0 \pm 28,98$ & $172,7 \pm 33,49$ & 0,9210 \\
\hline $3^{\prime}$ & $143,6 \pm 19,63$ & $147,3 \pm 29,01$ & 1,0000 \\
\hline $5^{\prime}$ & $127,0 \pm 18,25$ & $127,4 \pm 24,05$ & 0,9685 \\
\hline \multicolumn{4}{|c|}{ FC (bpm) } \\
\hline $1^{\prime}$ & $131,8 \pm 14,09$ & $116,4 \pm 15,76$ & $0,0249 *$ \\
\hline $3^{\prime}$ & $108,2 \pm 10,07$ & $96,45 \pm 10,79$ & $0,0159 *$ \\
\hline $5^{\prime}$ & $102,6 \pm 9,791$ & $94,09 \pm 10,10$ & 0,0576 \\
\hline \multicolumn{4}{|c|}{$\mathrm{DP}(\mathrm{mmHg} / \mathrm{bpm})$} \\
\hline $1^{\prime}$ & $22560 \pm 5208$ & $20223 \pm 5702$ & 0,2372 \\
\hline $3^{\prime}$ & $15609 \pm 2951$ & $14308 \pm 4149$ & 0,2003 \\
\hline $5^{\prime}$ & $13064 \pm 2441$ & $11982 \pm 2794$ & 0,2122 \\
\hline
\end{tabular}

Legenda: PAS: Pressão arterial sistólica; mmHg: milímetros de mercúrio; FC: frequência cardíaca; bpm: batimentos por minuto; DP: duplo produto e': minuto. 
DISCUSSÃO

Conforme exposto nos resultados, não foi observada diferença estatística significante em relação ao DP de tabagistas e não tabagistas. No entanto, o fato pode ser explicado pelo tempo de abstinência do GT.

Como explicado anteriormente, o cigarro aumenta a FC e a PA. Entretanto, após um período de abstinência, esses valores tendem a retomar seu estado normal. Os indivíduos do GT analisados nesse estudo estavam em abstinência tabagística por um período de 12 horas, o que influenciou no comportamento das variáveis hemodinâmicas necessárias para o cálculo do DP.

A abstinência tabagística implica em um conjunto de sinais e sintomas denominado síndrome de abstinência, que se inicia 8 horas após o último cigarro. Entre os sinais e sintomas, que são ansiedade, irritabilidade, insônia, apetite aumentado para doces, entre outros, estão a diminuição da FC e da PA ${ }^{16}$. Como, após fumar, a FC e PA ficam aumentadas, a tendência é que, durante a abstinência, essas variáveis retornem aos seus valores basais. Ainda, outros estudos observaram que tabagistas apresentam queda superior dos níveis pressóricos durante o sono, quando comparados a não tabagistas ${ }^{5}$.
Assim, pelo fato de as coletas terem sido realizadas no período da manhã e após as 12 horas de abstinência, os indivíduos do GT já estavam passando pelo processo da síndrome de abstinência, tendo, portanto, a redução a nível basal ou até inferior das variáveis hemodinâmicas coletadas para o cálculo do DP, o que justifica a redução dessa variável nesses indivíduos, não havendo, portanto, diferença estatística significante entre o GT e o GC.

No entanto, houve diferença estatística significante na FC durante o primeiro e terceiro minuto de recuperação, sendo a FC do GC de 131,8 $\pm 14,09$ e $108,2 \pm 10,07$, no primeiro e terceiro minuto, respectivamente, enquanto a do GT foi de $116,4 \pm 15,76$ e $96,45 \pm 10,79$, nos respectivos tempos. Desse modo, a FC do GT permaneceu mais baixa do que a do $\mathrm{GC}$ durante os 3 primeiros minutos de recuperação.

Isso pode ser explicado pelo fato de o indivíduo tabagista possuir respostas fisiológicas alteradas. Durante e após o exercício físico, essas respostas também se apresentam alteradas, devido aos efeitos deletérios causados pelo cigarro. Assim, pode-se observar que o GC apresentou a FC elevada quando comparado ao GT, o que se explica pelo fato de tabagistas apresentarem, 
habitualmente, uma menor resposta cardíaca frente ao exercício físico ${ }^{11}$.

\section{Conclusão}

Embora o cigarro cause aumento na frequência cardíaca, pressão arterial sistólica e, consequentemente no duplo produto, não houve diferença estatística significante entre o duplo produto de tabagistas e não tabagistas durante os cinco primeiros minutos de recuperação pós-esforço físico máximo.

\section{REFERÊNCIAS}

1. Brasil. Ministério da Saúde. Instituto Nacional do Câncer - INCA. Tabagismo: Dados e números.

2. Reichertl, J; Araujo, AJ; Gonçalves $\mathrm{CMC}$ et al. Diretrizes para Cessação do Tabagismo. J Bras Pneumol. 2004 Ago; 30(12)

3. Gauravi, AM; Sharmila, $A P$ and Surendra, SS. An overview of the tobacco problem in India. Indian J Med Paediatr Oncol. 2012;33(3):139-145. http://dx.doi.org/10.4103/0971-5851.103139

4. Oliveira, AM. Combate ao tabagismo: plano de intervenção em saúde no município de São Roque de Minas. Trabalho de Conclusão de Curso apresentado ao Curso de Especialização em Atenção Básica em Saúde da Família da UFMG. 2013.

5. Morillo, MG; Amato, MCM e Filha, SCP. Registro de 24 Horas da Pressão Arterial em Tabagistas e Não-Tabagistas. Arq Bras Cardiol. 2006;87:504-511. http://dx.doi.org/10.1590/S0066782X2006001700016

6. Hollmann, W; Hettinger, TH; Medicina de esporte: fumo e capacidade de desempenho físico. 1989.
7. Pureza, DY et al. Efeitos cardiovasculares da abstinência do fumo no repouso e durante o exercício submáximo em mulheres jovens fumantes. Ver Bras Med Esporte. 2007;13:292-296. http://dx.doi.org/10.1590/S151786922007000500003

8. Nogueira, KT; Fumo, CM e Silva, M. Tabagismo em adolescentes numa escola da rede pública do Estado do Rio de Janeiro. Adolescência \& Saúde. 2004.

9. Azoubel, R; Torres, GV; Silva, LWS; Gomes, FV e Reis, LA. Efeitos da terapia física descongestiva na cicatrização de úlceras venosas. Rev. esq. enferm. USP. 2010;44(4):1085-92.

10. Pessuto, J e Carvalho, EC. Fatores de risco em indivíduos com hipertensão arterial. Rev.latino-amer.enfermagem. 1998;6(1):33$39 . \quad$ http://dx.doi.org/10.1590/S010411691998000100006

11. Nunes, E. Consumo de tabaco. Efeitos na saúde. Rev Port Clin Geral. 2006;22:22544.

12. Souza, EN. Análise do efeito do treinamento de força de resistência associado ao exercício aeróbio nos parâmetros: composição corporal, cardiorrespiratório e neuromuscular. Dissertação de Mestrado apresentada ao Programa de Pós Graduação em Ciências Biológicas do Instituto de Pesquisa e Desenvolvimento da Universidade do Vale do Paraíba. 2003.

13. Polito, MD e Lemos, A. Comportamento do duplo-produto em diferentes posições corporais nos exercícios contra-resistência. Fit Perf J. 2003;2(5):279284.

14. Polito, MD e Farinatti, PTV. Respostas de frequência cardíaca, pressão arterial e duplo-produto ao exercício contra- 
resistência: uma revisão da literatura. Rev Port Cien Desp. 2003;3(1):79-91.

15. Brum, PC; Forjaz, CLM; Tinucci, T e Negrão CE. Adaptações agudas e crônicas do exercício físico no sistema cardiovascular. Rev. paul. Educ. Fís. 2004;18:21-31.

16. Marques, ACPR et al. Consenso sobre o tratamento da dependência de nicotina. Rev Bras Psiquiatr. 2001;23(4):200-14. http://dx.doi.org/10.1590/S1516-

44462001000400007

Recebido para publicação em 12/08/2014

Revisado em 28/08/2014

Aceito em 01/09/2014 